This article was downloaded by: [UOEH - Journal of Occupational and Environmental Hygiene]

On: 13 May 2009

Access details: Access Details: [subscription number 768371016]

Publisher Taylor \& Francis

Informa Ltd Registered in England and Wales Registered Number: 1072954 Registered office: Mortimer House, 37-41 Mortimer Street, London W1T 3JH, UK

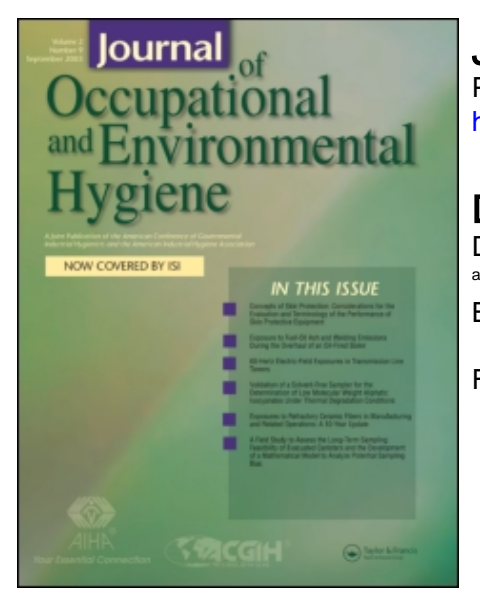

Journal of Occupational and Environmental Hygiene

Publication details, including instructions for authors and subscription information:

http://www.informaworld.com/smpp/title content=t713657996

\title{
Dust Control Effectiveness of Drywall Sanding Tools
}

Deborah E. Young-Corbett a; Maury A. Nussbaum ${ }^{b}$

a Department of Civil and Environmental Engineering, Myers-Lawson School of Construction, Virginia Tech, Blacksburg, Virginia ${ }^{b}$ Department of Industrial and Systems Engineering, Virginia Tech, Blacksburg, Virginia

First Published on: 01 July 2009

To cite this Article Young-Corbett, Deborah E. and Nussbaum, Maury A.(2009)'Dust Control Effectiveness of Drywall Sanding Tools',Journal of Occupational and Environmental Hygiene,6:7,385 - 389

To link to this Article: DOI: $10.1080 / 15459620902918852$

URL: http://dx.doi.org/10.1080/15459620902918852

\section{PLEASE SCROLL DOWN FOR ARTICLE}

\footnotetext{
Full terms and conditions of use: http://www.informaworld.com/terms-and-conditions-of-access.pdf

This article may be used for research, teaching and private study purposes. Any substantial or systematic reproduction, re-distribution, re-selling, loan or sub-licensing, systematic supply or distribution in any form to anyone is expressly forbidden.

The publisher does not give any warranty express or implied or make any representation that the contents will be complete or accurate or up to date. The accuracy of any instructions, formulae and drug doses should be independently verified with primary sources. The publisher shall not be liable for any loss, actions, claims, proceedings, demand or costs or damages whatsoever or howsoever caused arising directly or indirectly in connection with or arising out of the use of this material.
} 


\title{
Dust Control Effectiveness of Drywall Sanding Tools
}

\author{
Deborah E. Young-Corbett ${ }^{1}$ and Maury A. Nussbaum ${ }^{2}$ \\ ${ }^{1}$ Department of Civil and Environmental Engineering, Myers-Lawson School of Construction, Virginia \\ Tech, Blacksburg, Virginia \\ ${ }^{2}$ Department of Industrial and Systems Engineering, Virginia Tech, Blacksburg, Virginia
}

\begin{abstract}
In this laboratory study, four drywall sanding tools were evaluated in terms of dust generation rates in the respirable and thoracic size classes. In a repeated measures study design, 16 participants performed simulated drywall finishing tasks with each of four tools: (1) ventilated sander, (2) pole sander, (3) block sander, and (4) wet sponge. Dependent variables of interest were thoracic and respirable breathing zone dust concentrations. Analysis by Friedman's Test revealed that the ventilated drywall sanding tool produced significantly less dust, of both size classes, than did the other three tools. The pole and wet sanders produced significantly less dust of both size classes than did the block sander. The block sander, the most commonly used tool in drywall finishing operations, produced significantly more dust of both size classes than did the other three tools. When compared with the block sander, the other tools offer substantial dust reduction. The ventilated tool reduced respirable concentrations by $88 \%$ and thoracic concentrations by $85 \%$. The pole sander reduced respirable concentrations by $58 \%$ and thoracic by $50 \%$. The wet sander produced reductions of $60 \%$ and $47 \%$ in the respirable and thoracic classes, respectively. Wet sponge sanders and pole sanders are effective at reducing breathing-zone dust concentrations; however, based on its superior dust control effectiveness, the ventilated sander is the recommended tool for drywall finishing operations.
\end{abstract}

Keywords construction work, drywall, dust, dust control, ventilated sander

Address correspondence to: Deborah Young-Corbett, Virginia Tech, Civil and Environmental Engineering, Myers-Lawson School of Construction, 310B Bishop Favrao Hall, Blacksburg, VA 24061; e-mail: dyoung@vt.edu.

The contents of the article are solely the responsibility of the authors and do not necessarily represent the official views of the sponsor.

\section{INTRODUCTION}

$\mathrm{D}$ rywall finishing operations have been associated with worker overexposure to dust that contains known particulate respiratory health hazards. The National Institute for Occupational Safety and Health (NIOSH) conducted an evaluation of worker exposure to drywall dust and potential health effects associated with drywall finishing work during renovation activities. ${ }^{(1)}$ This study of 10 workers performing drywall-sanding tasks evaluated the main constituents of drywall joint compound, worker respiratory symptoms, and dust exposure levels. Drywall finishers were exposed to as much as 10 times the permissible exposure limit (PEL) of 15 $\mathrm{mg} / \mathrm{m}^{3}$ for total dust. The PEL for respirable dust, $5 \mathrm{mg} / \mathrm{m}^{3}$, was also exceeded. Respiratory symptoms were common among drywall finishers and tended to improve when workers were away from the workplace.

Joint compound constituents were identified as calcite, quartz (silica), talc, mica, gypsum, clays (attapulgite and kaolinite), and perlite. The two most prevalent constituents were calcite and mica. The term mica refers to a family of minerals of similar chemical composition and physical properties. These are potassium aluminum silicates with variable amounts of iron and magnesium. Mica has been associated with pneumoconiosis. ${ }^{(2)}$ Calcite, a form of calcium carbonate $\left(\mathrm{CaCO}_{3}\right)$ found in limestone, chalk, and marble, has been implicated in airway obstruction. ${ }^{(3)}$ Bohadana $^{(3)}$ found significant across-shift decline in all parameters associated with airway obstruction in chalk powder (calcite) manufacturing workers. Calcite exposure levels correlated with airway function decrement. Quartz (crystalline silica) has well-documented effects on the human respiratory system and has been associated with silicosis, ${ }^{(4)}$ a fibrotic disease of the lung, and malignant neoplasm of the lung. ${ }^{(5)}$ The International Agency for Research on Cancer (IARC) has classified quartz as a "group I human carcinogen," a classification indicating that sufficient evidence of causality exists in the scientific literature. ${ }^{(6)}$ Because the mineral dusts associated with drywall finishing work have been associated with diseases of both the upper and lower airways, the present study evaluated the generation of dust in both the thoracic and respirable size classes during the sanding task.

A study of exposure to silica dust in the U.S. construction industry found that "silica exposures are grossly unacceptable in the U.S. construction industry."(7) Personal sampling was performed for workers in the following trades: bricklayers, wall finishers, and laborers. Probabilities of overexposure were estimated to be between $64.5 \%$ and $100 \%$ for silica 
and between $8.2 \%$ and $89.2 \%$ for other dust. Rappaport et al. ${ }^{(7)}$ indicated that engineering and administrative controls are needed to reduce worker exposures. Silica exposures were significantly reduced by wet methods of dust suppression (300\% reduction in concentrations) and ventilated enclosures (600\% reduction in exposure concentration).

Engineering and personal protective equipment technologies exist for the control of worker exposure to dust from construction drywall finishing operations: ventilated sanding, wet sanding, pole sanding, and respiratory protection. Ventilated sanding technology involves a vacuum system attached to the sanding surface. Dust is collected at the point of generation and pulled into a collection basin. Wet sanding is accomplished in one of two methods. In one method, the drywall compound is allowed to cure and then rewetted prior to sanding. Rewetting is accomplished either by misting or wiping a damp sponge or cloth over the surface. A second method involves a sponge that has an abrasive coating surface. The sponge is moistened and used as a sanding block. This is the wet method employed in the current study. In pole sanding, the sanding surface is attached to the end of a pole, and the worker holds the other end while performing the operation. This reduces worker exposure to the dust by removing the worker from the point of dust generation.

A NIOSH Hazard Control Study ${ }^{(8)}$ found that vacuum sanding systems reduce drywall dust levels by $80 \%$ to $97 \%$. This evaluation of five commercially available ventilated drywall sanding systems found that four of the five reduced dust concentrations by greater than $90 \%$. NIOSH also recommends pole-sanding systems as a means of drywall dust reduction in a worker's breathing zone. The Association of Wall and Ceiling Industries (AWCI) recommends that finishers employ wet methods to reduce worker dust exposure. To date, no empirical evaluation of all of these recommendations has been published; therefore, the current study aimed to evaluate, in a controlled laboratory protocol, the relative dust generation rates of the NIOSH and AWCI-recommended sanding methods and the current industry standard sanding technique (dry sanding block).

\section{METHODS}

\section{Experimental Design}

In a repeated measures study design, participants performed a simulated drywall finishing task in a laboratory setting. Each participant performed the task four times, once with each sanding technology of interest. The four sanding tools were: (1) block, (2) ventilated, (3) pole, and (4) wet sponge sanders (Figure 1). Presentation order of the tools was balanced using a $4 \times 4$ Latin square design. Experimental protocols were reviewed and approved by the Institutional Review Board of Virginia Tech.

\section{Participants}

The 16 study participants were recruited from a population of university students. All were over the age of 18 and had no prior experience with drywall sanding tools. Because all pairwise comparisons among the four sanding technologies were of interest, calculation of the required number of participants was based on the Scheffé method of multiple comparisons at an overall confidence level of $95 \%$. The confidence interval was selected with the intent of indicating a difference in a pair of treatment combinations, if the true difference in total dust concentration levels is at least 3 $\mathrm{mg} / \mathrm{m}^{3}$. This value is $20 \%$ of the occupational exposure limit (OEL) established for particulates-not-otherwise-classified, a category that includes the dusts in this study. This is also the mean concentration found in the only existing prior study of drywall dust exposure assessments. ${ }^{(1)}$ In the NIOSH study, the standard deviation was 1.98 for drywall finisher dust exposures. From statistical power tables, ${ }^{(9)}$ with $\alpha=0.05$ and $\beta=0.20$, the number of required blocks (participants) was 16 .

\section{Laboratory Description}

A $4 \mathrm{ft} \times 4 \mathrm{ft}$ piece of drywall board was prepared for each participant. To each board were applied four strips of $0.95 \mathrm{~L}$ (4 cups) of drywall joint compound (Sheetrock Brand, Lightweight All Purpose Joint Compound; United States Gypsum Company, Chicago, Ill.). These strips were applied and allowed to cure, per manufacturer's instructions, by a trained drywall finisher. The strips were equal in width and length and were applied to the flat surface of a sheet of drywall. The board was attached to the wall inside a laboratory enclosure, at a fixed board center height of $1.68 \mathrm{~m}$, the 50th percentile U.S. adult height. There was no ventilation of the enclosure during the sanding tasks.

\section{Independent Variables}

Four drywall sanding tools, shown in Figure 1, were employed in this study: (1) a powered vacuum sander (model 7800; Porter Cable Inc., Cleveland, Ohio), (2) a block sander (3M Hand Sander; 3M, St. Paul, Minn.), (3) a pole sander (Gator model 7155; Ali Industries Inc., Fairborn, Ohio); and (4) a wet method (3M Sanding Sponge 180 grit). All four sanding tools had the same level of abrasiveness of the sanding surface (180 grit).

\section{Dependent Variable: Dust Concentration (Thoracic and Respirable)}

Two dependent variables, thoracic particulate concentration and respirable particulate concentration, were measured continuously throughout each sanding session. Concentration data were converted into delta dust concentrations, the change in concentration between sanding session start and end, and were employed in statistical analyses. Drywall joint compound dust concentrations were measured throughout the duration of each drywall sanding task session, using two DUSTTRAK Sidepak 

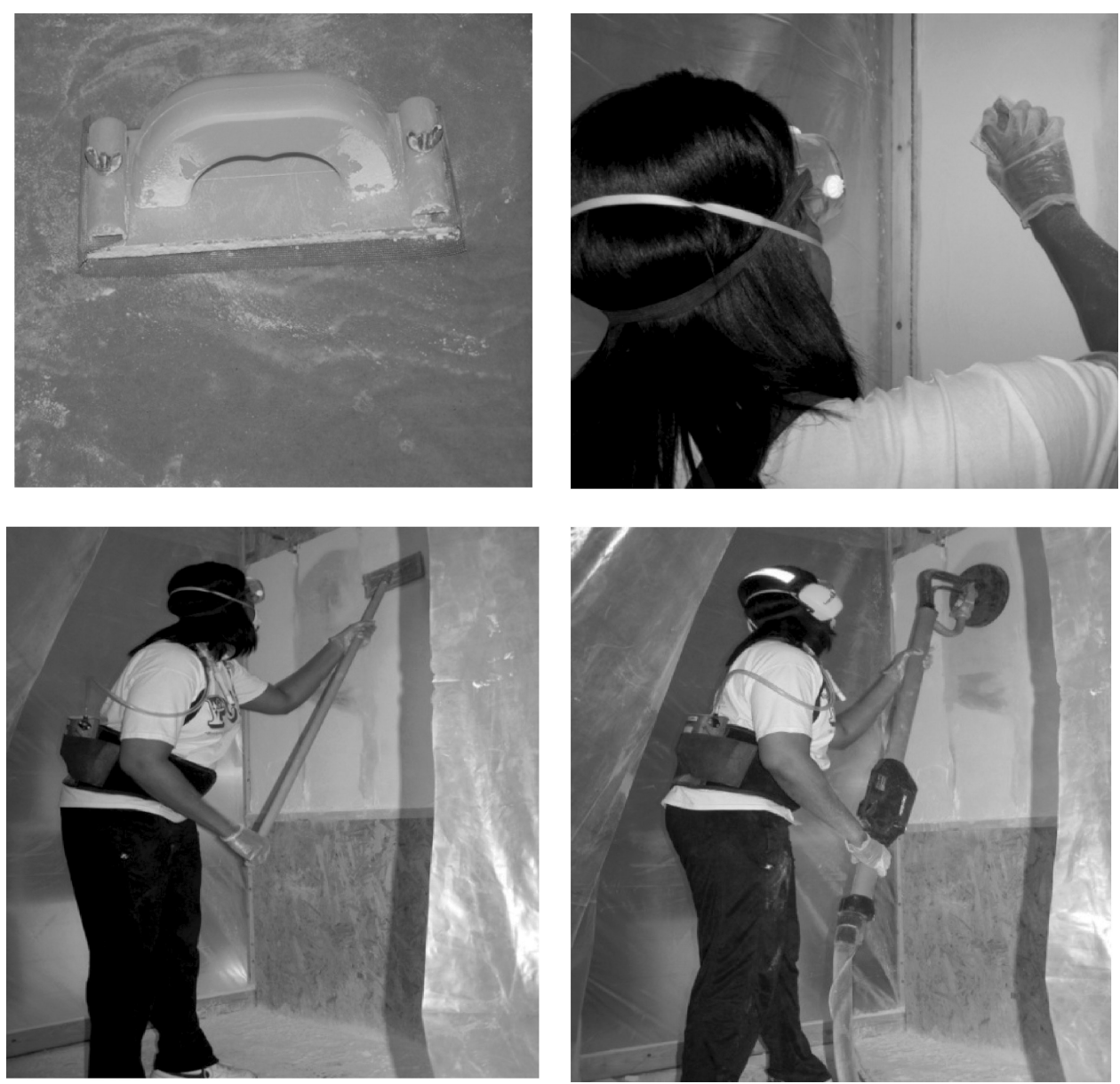

FIGURE 1. Sanding technologies: block hand sander, wet sanding sponge, pole sander, ventilated sander

AM510 Personal Aerosol Monitors (TSI Inc., Shoreview, Minn.). The AM510 is a miniature, battery-operated, laser photometer that measures airborne particulate matter in terms of mass concentration in units of milligrams per cubic meter of air sampled $\left(\mathrm{mg} / \mathrm{m}^{3}\right)$. Each participant wore two sampling trains involving the AM510 monitors: one that employed a 10-mm nylon Dorr-Oliver cyclone inlet (Gilian; Sensidyne, Clearwater, Fla.,) that had a median cut point of $4.0 \mu \mathrm{m}$ aerodynamic diameter and one that employed a TSI PM10 Impactor (Sidepak AM510 kit impactor) that had a median cut point of $10 \mu \mathrm{m}$ aerodynamic diameter. These two size classes were chosen because they represent the respirable and thoracic particulate size classes, respectively, that are implicated in the respiratory diseases associated with this occupational task.

The sampling train air inlet orifices were attached within a 1-ft radius of the participants' mouth and nose (i.e., the breathing zone). The sampling trains were mounted to a waist belt with suspenders, so that the positioning of the air inlets and AM510s would be consistent on all participants. Flow rates of $1.7 \mathrm{~L} / \mathrm{min}$ were used in both sampling trains and were calibrated before and after each experimental session, using a DryCal DC-Lite Primary Flow Meter (BIOS Inc., Butler,
N.J.). A 10-sec logging interval was chosen, and a calibration factor of 0.90 was used, since that is the manufacturer's recommended factor for the sampling of calcite dust, a primary constituent of drywall joint compound.

Ambient dust concentrations at the beginning of each sanding task session were measured and subtracted from any following measurements made during the sanding task sessions. The resulting delta concentrations were averaged to provide a 5-min, time-weighted average delta concentration (average delta concentration). This average delta concentration was the outcome measure used in the statistical analyses.

\section{Procedures}

Before participants entered the lab, several preparatory actions were undertaken. The AM510s were calibrated to ensure an airflow rate of $1.7 \mathrm{~L} / \mathrm{min}$ and were zeroed. The wallboard panels were placed on the wall mount in the experimental enclosure. Fresh sanding screens were installed on the block, pole, and ventilated sanding tools, and a fresh sanding sponge was prepared.

On arriving at the lab, participants reviewed and completed informed consent forms and received brief training on the use of protective equipment and an overview of the project 


\begin{tabular}{lcccc}
\hline & $\begin{array}{c}\text { Respirable } \\
\text { Delta } \\
\text { Concentration } \\
\text { Mean (SD) }\end{array}$ & $\begin{array}{c}\text { Respirable } \\
\text { Rank } \\
\text { Mean (SD) }\end{array}$ & $\begin{array}{c}\text { Thoracic Delta } \\
\text { Concentration } \\
\text { Mean (SD) }\end{array}$ & $\begin{array}{c}\text { Thoracic } \\
\text { Rank } \\
\text { Mean (SD) }\end{array}$ \\
\hline Ventilated sander $(\mathrm{n}=16)$ & $0.54(0.47)$ & $1.0(0.0)$ & $0.96(0.92)$ & $1.2(0.4)$ \\
Pole sander $(\mathrm{n}=16)$ & $1.93(1.09)$ & $2.6(0.5)$ & $3.13(1.99)$ & $2.5(0.9)$ \\
Block sander $(\mathrm{n}=16)$ & $4.65(2.32)$ & $4.0(0.0)$ & $6.31(3.20)$ & $3.9(0.3)$ \\
Wet sponge sander $(\mathrm{n}=16)$ & $1.85(1.15)$ & $2.4(0.5)$ & $3.35(2.37)$ & $2.3(0.5)$ \\
\hline
\end{tabular}

methods. They were then provided with a NIOSH-approved $\mathrm{N}-100$ filtering facepiece, goggles, gloves, earmuffs, and the sampling train harness. Participants were instructed on the use of the first of the four drywall sanding tools and instructed to sand one joint compound strip for $5 \mathrm{~min}$. They were given the goal of removing as much of the joint compound as possible during the 5-min period.

After reviewing task parameters for the trial and receiving training on the proper use of the first sanding tool, the participants entered the sanding chamber, the AM510s' recording sessions were initiated, and a timer was set for $5 \mathrm{~min}$. Participants sanded for the 5-min period and then the AM510s' dataloggings were paused while the participants completed a usability questionnaire for the tool just used. Results of that usability analysis were employed in a separate study by the authors. Participants then received training for the next sanding tool they were to use. Four sessions proceeded according to this protocol, until all tools had been used. The participants were compensated for their time.

\section{Analysis}

An examination of the data revealed that the distributions of respirable and thoracic dust concentrations were non-normally distributed. Therefore, the concentration data were converted to ranks and the Friedman Test was employed to test for effects of tool type on respirable and thoracic rank. Tukey's HSD was used to perform all post hoc comparisons of means. All statistical analyses were performed using JMP 8.0 (SAS Institute Inc., Cary, N.C.), with significance determined when $\mathrm{p}<0.05$.

\section{RESULTS}

\section{Dust Concentration}

Means and standard deviations for the delta dust concentrations and ranks are presented in Table I. Friedman's Test indicated significant differences between tool types for thoracic dust concentration $(\mathrm{F}(3,60)=59.37, \mathrm{p}<0.0001)$ and respirable dust concentration $(\mathrm{F}(3,60)=183.17, \mathrm{p}<0.0001)$. Subsequent pairwise comparisons of the means revealed that mean rank for the ventilated drywall sanding tool was significantly lower than the mean ranks of the other three tools. The mean ranks of the pole sander and wet method were significantly lower than the mean ranks for the block sander.

\section{DISCUSSION}

$\mathrm{T}$ he ventilated sander produced significantly less dust in the thoracic and respirable size classes than did all the other sanding technologies. The pole sander and wet sponge sander produced significantly less respirable and thoracic dust than did the block sander but more than did the ventilated sander. The block sander produced significantly more dust in both size classes than did the other three sanding tools. The ventilated sander reduced participant dust exposure by $88 \%$ in the respirable size class and $85 \%$ in the thoracic size class when compared with the block sander. The pole sander and wet sponge sander also produced substantial reductions in dust concentrations from that of the block sander. The block sander, the tool used most commonly in drywall finishing industrial practice, produced significantly more dust in both size classes compared with the other three technologies (Table II). Therefore, worker exposure to

TABLE II. Percent Dust Reduction When Compared with Block Sander

\begin{tabular}{lcc}
\hline Tool & Respirable & Thoracic \\
\hline Ventilated sander & 88 & 85 \\
Pole sander & 58 & 50 \\
Wet sponge sander & 60 & 47 \\
\hline
\end{tabular}

$$
\text { Dust reduction percentage }=\frac{(\text { Block sander mean concentration })-(\text { Other tool mean concentration })}{(\text { Block sander mean concentration })} \times 100
$$


drywall dust can likely be substantially reduced if ventilated, pole, or wet method techniques are used in practice, with ventilated sanders offering the greatest reductions of $85 \%$ to $88 \%$.

These findings are consistent with previously published NIOSH case study data ${ }^{(8)}$ that reported that ventilated sanders reduced worker exposure by $80 \%$ and $97 \%$, on separate trials. They are also consistent with the NIOSH Health Hazard Evaluation that suggested that pole sanders might have reduced worker exposure. ${ }^{(8)}$ Further, this study supports the AWCI recommendation of the wet method for dust control purposes. Based on the present results, a ventilated sander is the tool recommended by the authors for use in the drywall finishing industry. The pole sander and wet method, despite findings of their effectiveness in reducing dust concentrations, are not recommended. In a study of drywall sanding tool usability, both the pole sander and wet sponge sander performed poorly in terms of ease of use and perceived productivity. ${ }^{(10)}$

A recent study of industry usage rates of these technologies found that $15.9 \%$ of firms reported always using ventilated sanders, and $24.2 \%$ reported using them often. ${ }^{(10)}$ This finding indicates that nearly $60 \%$ of firms are not using the technology on a regular basis. Furthermore, these usage rate findings were likely artificially inflated. Open-ended, follow-up questions found that some respondents were referring to the use of shop vacuum cleaners for post-sanding dust removal, or to room ventilation fans, in answering that question. In all likelihood, far less than $40 \%$ of firms are using ventilated sanders on a regular basis. Therefore, in practice, worker exposures to particulate respiratory hazards could be reduced through increased use of ventilated sanding tools within the drywall finishing industry.

\section{LIMITATIONS}

$\mathrm{O}$ ne primary limitation stems from the laboratory nature of this study. While such studies are more robust regarding internal validity, they are weaker in terms of external validity. An additional limitation of the present study is the fact that the participants were novice users of all the drywall sanding tools under consideration. The decision to use novice user participants was carefully considered and deemed appropriate because of the importance of minimizing any confounding error arising from previous experience with drywall sanding technology.

The block sander is the most commonly used finishing tool in the drywall installation industry. The other three tools are used infrequently in practice. ${ }^{(10)}$ Therefore, it can be assumed that professional drywall finisher participants would have had variable proficiency with the four tools under study, which could have contributed bias to the findings. Future research is needed to ascertain the dust control effectiveness of these tools under actual occupational task conditions.

\section{CONCLUSION AND RECOMMENDATIONS}

$\mathrm{D}$ rywall finishing poles and wet sponge sanders can reduce worker exposure to dust. However, the ventilated sander is recommended for the control of worker exposure to dust during drywall finishing operations. This recommendation is made based on the significantly better performance in terms of reducing dust concentrations in the worker's breathing zone.

\section{ACKNOWLEDGMENTS}

$T$ his research was made possible through grant funding received from The Johns Hopkins University Occupational Safety and Health Education and Research Center of the National Institute for Occupational Safety and Health (JHU Regional NIOSH OSHERC).

\section{REFERENCES}

1. National Institute for Occupational Safety and Health (NIOSH), Hazard Evaluations and Technical Assistance (HETA): Health Hazard Evaluation Report 94-0078-2660, by A.K. Miller, E.J. Esswein, and J. Allen. NIOSH, 1997.

2. Skulberg, K.R., B. Gylseth, V. Skaug, and R. Hanoa: Mica pneumoconiosis: A literature review. Scand. J. Work Environ. Health 11:65-74 (1985).

3. Bohadana, A.B., N. Massin, P. Wild, and G. Berthiot: Airflow obstruction in chalk powder and sugar workers. Int. Arch. Occup. Environ. Health 68(4):243-248 (1996).

4. National Institute for Occupational Safety and Health (NIOHS), Silicosis and Silicate Disease Committee: Diseases associated with exposure to silica and non-fibrous silicate minerals. Arch. Pathol. Lab. Med. 112:673-720 (1988).

5. Dong, D., G. Xu, Y. Sun, and P. Hu: Lung cancer among workers exposed to silica dust in Chinese refractory plants. Scand. J. Work Environ. Health 21(Suppl 2):69-72 (1995).

6. International Agency for Research on Cancer (IARC): Monographs on the Evaluation of Carcinogenic Risks to Humans, Volume 77, Some Industrial Chemicals. Lyons, France: IARC, 2000.

7. Rappaport, S.M., M. Goldberg, P. Susi, and R.F. Herrick: Excessive exposure to silica in the US construction industry. Ann. Occup. Hyg. 47(2):111-122 (2003).

8. Mead, K.R., and A.K. Miller: Control of drywall sanding dust exposures. Appl. Occup. Environ. Hyg. 15:820-821 (2000).

9. Kutner, M.H, C.J. Nachsteim, and J. Neter: Solutions for Applied Linear Regression Models, 4th ed. New York: McGraw-Hill, 2003.

10. Young, D.E.: "Evaluation of Dust Control Technologies for Drywall Finishing Operations: Industry Implementation Trends, Worker Perceptions, Effectiveness and Usability." Ph.D. diss., Virginia Tech, Blacksburg, Va., 2007. Available at http://scholar.lib.vt.edu/theses/. Accessed August 9, 2007. 